\title{
Intégration financière et désintégration monétaire : La Turquie en crise de confiance
}

Faruk Ülgen

\section{(2) OpenEdition \\ 12 Journals}

Édition électronique

URL : http://journals.openedition.org/ei/308

DOI : 10.4000/ei.308

ISSN : 2553-1891

Éditeur

Association Économie et Institutions

Édition imprimée

Date de publication : 30 octobre 2007

Pagination : 67-98

ISSN : 1775-2329

\section{Référence électronique}

Faruk Ülgen, «Intégration financière et désintégration monétaire : La Turquie en crise de confiance », Économie et institutions [En ligne], 10-11 | 2007, mis en ligne le 31 janvier 2013, consulté le 10 décembre 2020. URL : http://journals.openedition.org/ei/308 ; DOI : https://doi.org/10.4000/ei.308

Revue Économie et institutions 


\section{Intégration financière et désintégration monétaire : La Turquie en crise de confiance ${ }^{1}$}

\section{Faruk Ülgen²}

\section{Introduction}

Le consensus de Washington, entérinant l'acception de l'optimalité des mécanismes de marché et la nécessité du retrait de l'Etat et des règlementations pour l'amélioration des structures économiques, a débouché dans les années 1990 sur une vague de libéralisation financière dans les pays émergents. Dans cette vague, la Turquie a procédé à des réformes de grande ampleur qui avaient déjà débuté au début de 1980. Mais, connaissant des déséquilibres internes et externes durables, elle a subi une crise majeure en 1994 et a réitéré ses choix de politique économique qui l'ont amenée à la première crise du XXIe siècle en 2000-2001.

Dans cet essai, cette évolution est analysée en utilisant une grille de lecture spécifique qui questionne la pertinence du régime monétaire fondé sur une libéralisation financière étendue et qui considère ces crises comme des crises de la confiance que doivent inspirer les règles monétaires en vigueur. Plus précisément, notre argumentaire suit les enseignements des développements récents de l'approche monétaire qui ont permis de préciser le rapport à la souveraineté de la monnaie, considérée comme institution de coordination fondatrice d'une économie de marché décentralisée.

En effet, en économie, les débats sur la nature de la monnaie, sur les règles monétaires et, par conséquent, sur les politiques monétaires optimales, n'échappent pas à l'opposition entre deux visions radicalement différentes : l'approche monétaire et l'approche réelle. L’approche monétaire considère la monnaie comme catégorie économique première dans l'appréhension des phénomènes économiques ${ }^{3}$ tandis que l'approche réelle ${ }^{4}$, fondée sur la croyance

1 Je remercie les deux rapporteurs anonymes de la revue et le Professeur Jaime Marques Pereira pour leurs remarques et suggestions qui m'ont permis d'améliorer sensiblement une version antérieure de ce texte. Les erreurs et omissions relèvent toutefois de ma seule responsabilité.

2 Université Grenoble 2, CREPPEM, Ulgen.Faruk@upmf-grenoble.fr

3 Il existe de nombreux modèles qui se réclament de l'approche monétaire mais qui peuvent être très différents les uns des autres. Toutefois, ils ont comme point commun de chercher à expliquer le fonctionnement des économies de marché à partir de la monnaie. Pour une présentation critique de ces modèles, voir Ülgen (1995).

Economie et Institutions $-\mathrm{n}^{\circ} 10$ et $11-1$ er $\& 2$ e semestre 2007 
de l'existence des mécanismes de rééquilibration spontanée des marchés, considère la monnaie fondamentalement neutre, au moins à long terme.

A travers un exposé des fondements conceptuels de ces approches alternatives, cet article montre comment cette opposition méthodologique peut conduire à des conceptualisations différentes du système monétaire et de l'efficacité de la politique monétaire. On se borne ${ }^{5}$ ici à préciser l'intérêt de la conceptualisation en termes de confiance et de souveraineté monétaires pour appréhender la question de la stabilité monétaire et financière comme alternative au concept de crédibilité de l'action des autorités, découlant de l'approche réelle de l'équilibre économique général. Dans cette dernière, la crédibilité est restreinte à un problème de transparence et de responsabilité des politiques monétaires et des dépenses publiques restrictives. Cette position est principalement fondée sur le paradigme des Nouveaux Classiques (NEC) visant à garantir la neutralité de la monnaie. Une telle problématique fait l'impasse sur la question de la légitimité des règles en vigueur et surtout sur celle de la confiance en le régime monétaire. En effet, le paradigme NEC renvoie la crédibilité à la gestion conservatrice de la monnaie à travers une banque centrale isolée des considérations politiques. L'approche des Nouveaux Keynésiens (NK) refonde cette notion sur une règle de discrétion contrainte. Sous l'hypothèse de marchés imparfaits et à condition d'éviter les comportements temporellement incohérents, elle considère nécessaires les interventions discrétionnaires et aboutit à la rhétorique d'un Etat dit market friendly.

La thèse défendue dans ce travail est que la capacité de la politique économique à prévenir les crises monétaires et financières et à éviter la réduction de la croissance est tributaire de la confiance en la viabilité du régime monétaire. Les crises observées dans les pays émergents peuvent être considérées comme la manifestation

\footnotetext{
${ }^{4}$ Les modèles néoclassiques, monétariste, nouveaux classiques, etc.

5 Cette simplification est principalement liée à des contraintes de taille d'un article. Il convient néanmoins de préciser que ce choix est l'aboutissement de la réflexion sur la monnaie de nombreux travaux qui se situent dans la lignée de l'approche monétaire et qui cherchent à mieux cerner sa nature institutionnelle et " communicationnelle " à partir des résultats de recherche d'autres sciences sociales sur la question, comme la sociologie et l'anthropologie de la monnaie. Ces résultats vont au-delà de l'opposition standard entre l'autorégulation des marchés et la régulation politique hors marché, observée dans les modèles économiques alternatifs d'obédience néoclassique ou post keynésienne. Sur la portée d'un tel élargissement et sur les contraintes qu'il est susceptible d'impliquer dans l'analyse de la monnaie, nous nous permettons de renvoyer, entre autres, à Cartelier (2008).
}

68 Economie et Institutions - n 10 et $11-1$ er \& 2e semestre 2007 
des limites d'une conception restreinte de la crédibilité du fait d'omettre la question de la légitimité des politiques suivies ou de la considérer résolue dans l'application de la règle de politique monétaire anti-inflationniste. Or, l'efficacité des actions des institutions et de l'Etat est intimement liée au problème de l'intégrité du système de paiements. L'intégrité du système peut être définie comme la validité des règles générales qui déterminent les conditions d'émission, d'utilisation et d'annulation des dettes qui circulent dans l'économie sous forme monétaire. Par conséquent, la confiance en l'intégrité du système porte sur la souveraineté des règles monétaires et renvoie à la question de leur légitimité. De ce point de vue, il semble judicieux de prendre en compte, dans l'analyse des crises monétaires et financières, le problème de la gestion collectivement pertinente de la confiance en la monnaie qui se cristallise dans l'institutionnalisation des politiques monétaires. La stabilité monétaire et financière apparaît alors comme un résultat de la confiance que procure la légitimité de règles monétaires faisant l'objet d'un consensus social/politique permettant ainsi que les transactions économiques et le système de paiements soient considérés comme soutenables. Comme l'établissement d'un tel consensus et la stabilité macroéconomique se révèlent intimement liés, les politiques monétaires conservatrices (purement restrictives) ne sont pas une garantie de stabilité. Ceci souligne aussi que le bon fonctionnement du système monétaire -que seul doit garantir dans la pensée néoclassique le respect des équilibres monétaires et budgétaires- pose la question du consensus politique qui le fonde ${ }^{6}$.

Cette approche nous conduit ici à un diagnostic ambivalent des succès de la stabilisation de l'économie turque. Les principaux faits stylisés de la dynamique économique turque depuis les années 1980 montrent une longue évolution vers la faillite de l'Etat et du système bancaire à travers l'analyse des comportements des acteurs et de l'effritement de la confiance en le régime monétaire suivi. Il

6 Cette proposition théorique explicite la portée macroéconomique de la conception de la monnaie comme fait social total, une idée de Mauss que théorisent différents modèles (comme, par exemple, Cartelier (2008) et Théret (2008b)). En synthèse, il convient de souligner que, dans cette conception de la monnaie, la dimension proprement politique de la politique monétaire ne peut être "neutralisée ", comme le croît la pensée néoclassique, car les règles constitutives d'un système de compte et de paiement (convertibilité des dettes et émission des moyens de leur règlement) ne paraîtront crédibles et donc soutenables que dans la mesure où elles paraissent également légitimes. Cet article illustre la portée de cette thèse pour mieux cerner la question des sources de crises financières dans les économies émergentes (mais aussi dans les pays développés comme en témoigne la surprise de la crise des prêts hypothécaires aux Etats-Unis) que la théorie néoclassique n'a manifestement pas aidé à prévoir.

Economie et Institutions $-\mathrm{n}^{\circ} 10$ et $11-1$ er $\& 2$ e semestre 2007 
apparaît que la stabilisation ne dépend pas seulement du taux de change et de la politique monétaire restrictive, mais d'un consensus national et international sur les conditions de coexistence économique et politique et sur le régime monétaire choisi. L'aptitude de l'Etat à jouer un rôle d'arbitre dans la résolution des crises et dans la refondation de la structure productive délimite le domaine des possibilités pour retrouver une légitimité politique et détermine si la souveraineté monétaire et la stabilité macroéconomique peuvent être recouvrées ou non.

Cet article comporte deux sections. La première section expose d'abord la grille de lecture suivie. La stabilité monétaire est considérée à travers trois catégories de confiance qui déterminent le niveau de validité collective des règles de légitimation du régime monétaire. Les catégories de confiance vis-à-vis du système de paiement et de souveraineté monétaire sont évaluées afin de spécifier leur portée explicative; le fait que le consensus politique constitutif du régime monétaire -sa solidité ou sa fragilité- soit susceptible d'engendrer ou non une stabilité monétaire. Sur cette base, on procède ensuite à une évaluation du paradigme dominant (NEC mais aussi NK) soulignant qu'il ne permet qu'une analyse en termes d'adhésion de l'opinion financière et non de légitimité puisque cette dernière se pose par rapport à l'ensemble de la société. Cette adhésion de l'opinion financière est supposée représentative des comportements de l'ensemble des agents, ce qui rend une telle vision équivoque dans la mesure où elle ne permet pas de prévoir le retournement des anticipations et réduit de ce fait sa portée explicative à la fois normative et positive vis-à-vis des effets macroéconomiques des règles monétaires en vigueur sur les confiances et le consensus politique. La deuxième section expose brièvement l'évolution de l'économie turque depuis le début, en 1980, des réformes de libéralisation jusqu'au début des années 2000. Suivant l'analyse des catégories de la confiance, elle donne une interprétation des dynamiques de la crise de 2000-2001 en termes de viabilité du système de paiements à l'égard de la problématique de la confiance qu'inspire le régime monétaire. Une extrapolation de cette interprétation est ensuite proposée afin de considérer la trajectoire que l'économie turque suit sur la période 2002-2007.

\section{Stabilité monétaire}

Suivant la conception monétaire de l'économie adoptée dans ce travail, la capacité du régime monétaire à être viable dans le temps dépend de l'état de trois formes de confiance: hiérarchique, méthodique et éthique. (Aglietta et Orléan, 1998, 2004). Ceci nous permet de proposer une interprétation des conditions de stabilité

70 Economie et Institutions - n ${ }^{\circ} 10$ et $11-1$ er $\& 2$ e semestre 2007 
monétaire sensiblement différente de celle de crédibilité employée dans les paradigmes NEC et NK.

\section{1. La monnaie et la confiance en le régime monétaire}

Cette analyse se situe dans la lignée des approches monétaires ${ }^{7}$ dans lesquelles la monnaie est présentée comme un ensemble de règles qui constituent un processus de coordination sociale compatible avec les mécanismes de marché décentralisé. La monnaie est le moyen de paiement et de règlement nécessaire dans une économie dynamique où des soldes non nuls apparaissent continuellement à la fin de chaque série de transactions et où l'équilibre n'est pas donné préalablement aux échanges effectifs.

La monnaie a une dimension à la fois sociale (règles et institutions compatibles avec le marché), supra individuelle, et privée en tant que moyen d'action individuelle autorisant les rencontres et négociations entre agents séparés (Cartelier, 1998). Les règles communes qui définissent, délimitent et valident le système de paiements ne sont pas individuelles mais instituées, s'imposant à tous les individus. Dans ces règles prennent place l'existence et les modalités de fonctionnement d'une banque centrale arbitre, réputée extérieure au système, et des institutions monétaires (les banques) et financières dont le principe organisateur dépend d'une relation verticale fondée sur une hiérarchie : "la monnaie suppose dans sa construction la référence hiérarchique à l'autorité supérieure tout en étant égalitaire dans le principe de son usage " (Aglietta et al. 1998, p. 11). Cette structure, dont une définition spécifique correspond à la notion de régime monétaire, permet un lien social entre les différentes périodes, i.e. une continuité dans les relations de paiement. Par conséquent, la stabilité monétaire dépend des conditions de reproduction ou de permanence du système de paiements (Aglietta et Cartelier, 1998), conditions qui sont liées aux modalités de légitimation de l'institution monétaire.

La banque centrale, extérieure aux relations privées de paiement, établit la convertibilité entre des émetteurs distincts et la circulation routinière des signes monétaires permettent aux règles d'avoir une validité générale dans la communauté de paiements. La spécification première de la monnaie, l'unité de compte (Keynes, 1965), donne la forme quantitative aux relations entre agents économiques. Elle est intimement liée à l'idée de souveraineté en tant qu'attribut du pouvoir politique qui intervient dans la régulation du système de paiement à travers les vicissitudes du marché (Aglietta et Cartelier, 1998). L'autorité monétaire est l'ensemble des valeurs collectives, des normes qui coordonnent les comportements et

7 Voir Cartelier (2008) et Ülgen (1995), entre autres. 
décisions individuelles et au nom desquelles la cohésion d'une économie est affirmée. La crise monétaire peut alors être interprétée comme une crise de confiance en le régime monétaire à travers une fragmentation du système de paiement par différenciation des unités de compte selon les réseaux de dette différents dans un même espace monétaire.

La confiance qu'inspire le régime monétaire peut être identifiée à travers trois formes spécifiques mais interdépendantes: la confiance hiérarchique, la confiance méthodique et la confiance éthique.

La confiance dans la monnaie est ancrée sur sa capacité à assurer une coordination générale entre les agents, ce qui nécessite le maintien d'une régulation des conditions d'accès, de circulation et de remboursement des dettes/moyens de paiement (Ülgen, 1995). La monnaie ne remplit ce rôle que si elle est en même temps adossée à un compromis/un consensus social. La confiance hiérarchique, qui est fondée sur la stabilité de l'unité de compte et sur la garantie des moyens de paiement par une instance supérieure, la banque centrale, cristallise ce consensus (Théret, 2008b). La banque centrale est distincte des relations interindividuelles et assure la convertibilité de tous les signes monétaires en une unité de compte, mais elle se trouve contrainte elle-même par les règles dont elle est censée faire observer le respect par les intervenants. Cette forme de confiance est liée à la question de la souveraineté. Les instances de la souveraineté représentent les règles objectives et doivent être perçues extérieures aux relations privées. Elles jouent le rôle du tiers médiateur qui doit réguler les conflits latents ou exprimés sur les marchés pour la survie du système. Comme la reproduction de la société est supposée fondée sur la possibilité de circulation générale de la monnaie dans son rôle de coordinateur économique et de cycle ininterrompu de paiements, la validité des règles monétaires est liée à la souveraineté (Orléan, 2006, Théret, 2008b).

La confiance méthodique est fondée sur la continuité dans le temps des pratiques routinières. Cette continuité signifie l'acceptation générale de la monnaie, établie sur la confiance que les règles monétaires sont objectives et constituent des repères pour les actions futures: "Le système de paiements, conçu comme un ensemble de règles gérant les modalités, la temporalité et la validité des transactions monétaires, constitue donc la condition permissive de l'émergence d'une dynamique sociale routinière " (Scialom et Zlotowski, 1994, p. 715). Il faut alors que les relations de paiements, dans la répétition des actes privés, mènent les échanges à bonne fin pour que les agents considèrent leur communauté de paiements comme utile et nécessaire pour leurs objectifs. Lorsque la confiance méthodique est ébranlée, le consensus s'interrompt et la validité du régime monétaire est remise en question.

72 Economie et Institutions - n 10 et $11-1$ er \& 2e semestre 2007 
Néanmoins, la seule confiance méthodique ne permet pas de faire face aux situations de doute généralisé. L'institution des règles monétaires -au fondement de la confiance hiérarchique- doit intervenir en suspendant certaines règles du marché en vue de corriger les hésitations de la confiance méthodique et d'éviter une panique systémique qui se présente sous la forme d'une méfiance collective vis-à-vis des règles monétaires, constitutives du système de compte et de paiements. L'intervention du prêteur en dernier ressort transforme les dettes privées en dette sociale pour contrecarrer la défaillance généralisée du système de paiements. Mais cette intervention doit être vue par la communauté comme l'exercice non abusif de l'autorité hiérarchique de façon à ne pas créer une défiance des créanciers. Eviter cette défiance dont une forme spécifique est le sentiment d'aléa moral, ne passe donc pas par la disparition de la garantie du prêteur en dernier ressort, ce qui supprimerait la nécessaire confiance hiérarchique, mais par l'établissement des conditions dans lesquelles les agents en difficulté peuvent être ou non soutenus (Bagehot, 1873). Là est la sensibilité extrême du système de paiements qui relève de la confiance éthique.

Dans les économies modernes, la confiance éthique est fondée sur le principe de supériorité de l'individu sur le tout social. L'autorité hiérarchique doit veiller à ce que la valeur économique des contrats privés soit respectée et la reproduction des droits et des devoirs individuels soit assurée dans le temps à travers le respect des règles monétaires protégeant les droits des créanciers et établissant les obligations des débiteurs. Lorsque la confiance éthique disparaît, elle supprime aussi la confiance hiérarchique. Par exemple, dans les périodes où la dette sociale devient très élevée à l'égard des limites de la croyance des marchés aux interventions de l'autorité, les modalités de financement de la dette créent des conflits. Les périodes de forte inflation sont une traduction du refus des marchés de financer la dette sociale. La défiance à l'égard de l'institution monétaire se transforme en une méfiance éthique et en un mouvement spéculatif contre la monnaie nationale et les agents fuient la monnaie au profit des monnaies allogènes. Le consensus social, ainsi ébranlé, ne permet plus de continuer les relations monétaires et la confiance en la viabilité du régime s'effrite.

Une des solutions proposées aujourd'hui pour assurer la stabilité monétaire est d'établir la stabilité des prix comme objectif ultime. Ceci détermine la matrice d'action des banques centrales et crée une valeur normative dont la validité est renvoyée à un impératif éthique (Aglietta et Cartelier, 1998). Toutefois, cette solution considère le problème comme provenant uniquement d'une erreur de politique économique laxiste. Ainsi, lorsque la garantie des créanciers est établie sur la seule règle conservatrice, la crédibilité est réduite à une question d'éthique sans permettre pour autant de 
restaurer un consensus social sur les confiances ${ }^{8}$ dans la mesure où la question de la reproduction des règles monétaires dans le temps est renvoyée au seul critère de crédibilité ex ante fondée sur une norme de politique monétaire exogène.

L'appréhension des crises de confiance peut donc être liée aux conceptions particulières de la monnaie et de la souveraineté qui déterminent l'acceptabilité (ou le dysfonctionnement) des états (institutionnalisé, incorporé et objectivé) de la monnaie par laquelle s'institue (ou se disloque) la communauté de paiement comme forme politique (Marques-Pereira, 2006) et à travers laquelle les trois formes de confiance sont mises à l'épreuve. L'état institutionnalisé de la monnaie correspond aux règles qui définissent l'unité de compte et les instruments de paiement et institue un espace monétaire. L'état incorporé de la monnaie comme étalon de valeur lui permet d'être utilisée comme unité de mesure dans les opérations économiques. Son état objectivé en fait un moyen de paiement matérialisé dans les instruments de paiement acceptés. L'état incorporé de la monnaie sous-tend la confiance éthique se matérialisant dans sa capacité à assurer la coordination des transactions par l'acceptation du système de compte. Celle-ci va de pair avec une régulation de l'émission des moyens de paiements qui assure le règlement des dettes et la possibilité d'en contracter de nouvelles, ce qui détermine les conditions monétaires de reproduction sociale. Il en découle que la monnaie incorporée et la monnaie objectivée ne sont pas envisageables comme simples moyens d'un ordre spontané de marché à la Hayek. Ces états sont inséparables de l'état institutionnalisé de la monnaie qui établit l'unicité du système de compte (Théret, 2008a). On remarque ainsi que le risque de fragmentation est inhérent à la coexistence d'un système de compte unique et d'une pluralité de moyens de paiement dont le caractère fiduciaire peut être variable. La possibilité de faillites bancaires, résultant de la différence de qualité des créances composant l'actif bancaire, n'est enrayée que par la garantie de la banque centrale représentant la confiance hiérarchique. Le risque de crise monétaire peut se transformer en une crise de confiance éthique lorsque la hiérarchie des formes d'endettement et les compromis sociaux qui la fondent sont contestés. On est donc en permanence dans une société de conflits potentiels dont le dénouement économique pacifique

${ }^{8}$ Les trois niveaux de confiance peuvent être rapprochés des analyses d'Arestis et al. (2003), qui relient l'instabilité financière observée dans les pays en développement au faible développement des normes de confiance et des structures institutionnelles, et de Minsky (1996), qui décompose la structure institutionnelle en trois catégories: les autorités qui édictent les règles (confiance hiérarchique), les institutions et usages qui forment la routine des actions sur les marchés (confiance méthodique) et les administrations qui contrôlent l'application des règles (normes éthiques).

74 Economie et Institutions - n 10 et $11-1$ er \& 2e semestre 2007 
dépend de la validité permanente des normes collectivement entérinées en tant que repères pertinents et la politique monétaire est destinée à déterminer les modalités de conservation de l'intégrité du système de paiement.

\section{2. Dette, confiance et souveraineté}

La souveraineté est l'élément central qui doit réaffirmer la légitimité des normes collectives qui constituent l'ordre monétaire. Si l'ordre est légitime, la confiance méthodique est garantie par la confiance hiérarchique (Théret, 2008a). Il paraît alors utile de s'interroger sur l'interdépendance entre la souveraineté politique, déterminant une organisation particulière du système de paiement, et la souveraineté monétaire. L'analyse des crises comme une remise en cause de la légitimité monétaire fait ressortir une différenciation des usages spécifiques des états de la monnaie. Les crises internes se manifestent par une dégradation des confiances méthodique et hiérarchique et remettent en cause la légitimité de la souveraineté monétaire, c'est-à-dire les règles de monnayage. Dans les crises externes, le régime monétaire est mis sous tension par la concurrence d'une monnaie étrangère parce que l'Etat cherche ainsi à pallier son incapacité d'assurer la confiance éthique en sa monnaie. Les crises externes remettent simultanément en cause la souveraineté monétaire et la souveraineté politique car l'unité du système de compte est contestée. La perte de confiance éthique en la monnaie signifie alors une crise de souveraineté politique (MarquesPereira, 2006) ${ }^{9}$.

Plus généralement, à partir des programmes de libéralisation, l'unité d'un système de compte qui différencie la mesure de la valeur des actifs, des biens et du travail devient problématique puisque la confiance méthodique en la monnaie nationale est focalisée sur sa convertibilité externe. L'état incorporé de la monnaie que permet la routine des paiements internes dépend de la balance des paiements externes, donc d'une routine financière dont le point de mire devient le service de la dette publique, son encours et l'échéance des nouvelles émissions. Leur anticipation est centrée sur l'observation du taux d'intérêt directeur et du risque de change que celui-ci doit couvrir. C'est là un processus de décisions à la fois privées et publiques. Les décisions privées les plus déterminantes concernent l'acceptation ou non des prix d'offre et des clauses contractuelles des bons du Trésor qui sont la référence de la fixation des niveaux d'offre

9 Par exemple, en raison des difficultés à réguler divers conflits distributifs, l'Etat ne parvient à préserver l'unité du système de compte qu'en institutionnalisant sa fragmentation par une pluralité de moyens de paiement, ce qui menace l'inscription territoriale de la confiance éthique.

Economie et Institutions $-\mathrm{n}^{\circ} 10$ et $11-1$ er $\& 2$ e semestre 2007 
et de prix par ceux qui disposent d'un pouvoir de marché. Les décisions publiques doivent ajuster le besoin de financement externe (soldes courant et du capital) aux capacités de financement externe. La qualité des décisions des autorités est évaluée en permanence par les réactions des bailleurs de fonds internationaux et par les conditions d'ancrage de la monnaie nationale sur des repères extérieurs.

Lorsque la fragmentation du système de compte entre instruments de paiement débouche sur des niveaux élevés d'inflation, l'autorité change la règle monétaire. Elle peut mettre en place de nouveaux états objectivés de la monnaie suivant, par exemple, la contrainte de stabilité des prix que permettent les rigueurs monétaire et budgétaire, assorties des réformes structurelles fondées sur l'idée d'efficacité de l'allocation des ressources par l'autorégulation des marchés suivant le paradigme NEC. La confiance hiérarchique en la monnaie est rétablie par la reconnaissance des nouvelles valeurs de gouvernement, devenues aujourd'hui universelles, qui refondent l'idée de la souveraineté politique. L'importation de la monnaie internationale par l'ancrage du change permet la recentralisation du système de compte. Lorsque le taux de croissance économique s'élève, la pertinence de ces valeurs semble confirmée sans toutefois une déconcentration des revenus, ce qui peut rendre, à moyen terme, les règles monétaires fragiles dans la restauration des trois niveaux de confiance.

Les instabilités observées dans certains pays émergents, comme la Turquie et l'Argentine, montrent la difficulté pour les règles monétaires en vigueur de refonder la confiance d'une manière cohérente et durable. Cette difficulté se manifeste surtout dans la séquence d'essais infructueux de restaurer la confiance en la monnaie dans le temps puisque nombre de crises deviennent récurrentes et revêtent une forme endémique. En général, les politiques sont fondées sur un usage fonctionnel des états de la monnaie pour gérer la répartition par un endettement public qui impose une fragmentation du système de compte. Sur le plan de la confiance éthique, le paradigme NEC propose, comme ancrage pertinent, le système de prix relatifs, supposé garantir l'équilibre économique et définir l'espace d'exercice monétaire de la souveraineté politique. La règle de monnayage (sur le modèle monétariste) et les principes de gestion énoncés (un objectif quantitatif monétaire ou d'inflation, la discrétion contrainte ou des règles d'indépendance de la banque centrale) en constituent les modalités envisagées, observables, par exemple, dans la tentative de restauration de la cohérence du régime monétaire par un ancrage du change sur des références monétaires extérieures. Ainsi, la contrainte de la confiance hiérarchique est réduite dans l'approche NEC à la gestion conservatrice de la monnaie par des règles anti-

76 Economie et Institutions - n 10 et $11-1$ er \& 2e semestre 2007 
inflationnistes tandis que le consensus NK considère une discrétion contrainte. Mais la question de la pertinence des choix à l'égard de la viabilité du régime monétaire échappe à ces deux visions dans l'analyse des crises récurrentes.

\section{3. Crédibilité restreinte et économie monétaire}

La notion de crédibilité utilisée dans le paradigme NEC semble d'une portée restreinte à l'égard des caractéristiques fondamentales d'une économie monétaire dans laquelle la stabilité temporelle des relations économiques dépend principalement de la validité générale des règles monétaires qui permettent les rapports entre les différents acteurs (publics et privés) sur les marchés. Ces règles définissent, outre les espaces et modalités d'action des agents privés, un régime monétaire spécifique qui caractérise les politiques monétaires envisagées, leurs objectifs ainsi que les moyens qui seront mis en œuvre dans leur accomplissement.

A partir des années quatre-vingts, les régimes monétaires administrés et discrétionnaires s'ouvrent aux mécanismes d'arbitrage des marchés de plus en plus libéralisés. Le paradigme NEC (Lucas, 1972, Sargent et Wallace, 1975), fondement conceptuel des choix qui ont guidé cette évolution, détermine la stabilité des prix comme le seul objectif pertinent d'une politique monétaire responsable. L'efficacité des politiques est évaluée en termes de crédibilité, déduite des travaux sur l'incohérence temporelle des politiques économiques (Barro et Gordon, 1983). La crédibilité est définie comme la possibilité pour les autorités de convaincre les marchés qu'elles respecteront la politique monétaire annoncée, sous l'hypothèse particulière que les agents formulent des anticipations rationnelles. En adoptant une politique monétaire de rigueur, les autorités sont supposées développer une réputation, devenir crédibles et l'inflation se réduire sensiblement (McCallum, 1997). La crédibilité est alors déterminée à partir du critère de conformité des politiques suivies à une norme de politique économique qui dérive d'un référentiel $e x$ ante qui est donné à la fois par la règle de monnaie neutre et par le principe d'indépendance de la banque centrale (et donc de la politique monétaire). Or, la stabilisation monétaire dépend à la fois de la perception des réformes par les agents et de leur application dans le temps dans la mesure où l'obtention d'une crédibilité acceptée unanimement dépend d'un effet d'apprentissage intériorisé par les agents économiques.

Dans cette perspective, il convient de considérer, d'une part, la pertinence des politiques élaborées (apparaissent-elles légitimes à l'égard des déséquilibres subis ?) et, d'autre part, la façon dont les réformes sont appliquées et suivies (leur évaluation par les agents). En ce qui concerne la pertinence des politiques, la vision NEC 
renvoie à une règle unique : crédibilité anti-inflationniste par une gestion austère de la monnaie. Le choix revêt un aspect technique dont la légitimité est supposée liée, en termes normatifs, à l'établissement d'une instance à même d'appliquer la règle de neutralité de la monnaie. Il semble alors judicieux de remarquer que supposer qu'une règle de stabilité (ciblage d'inflation ou réputation conservatrice de la banque centrale) est légitime revient à présupposer une rationalité des agents conforme à la théorie ${ }^{10}$, ce qui revient à faire l'impasse sur la complexité de la légitimité politique qui ne peut être pourtant pensée que dans un cadre d'interdépendances entre plusieurs niveaux de confiance et de consensus dont cherchent à rendre compte les analyses politiques et sociologiques de 1'Etat ${ }^{11}$. Quant aux modalités d'application des réformes, ce paradigme explique les résultats médiocres par le manque de transparence et de responsabilité des autorités dont les comportements dévieraient des règles annoncées. Les marchés l'anticipant, la crédibilité est remise en cause et les attaques spéculatives se déclenchent. Mais dans ce cas, il n'est posé qu'un problème politique de résistance d'intérêts particuliers, ce qui justifierait l'indépendance de la banque centrale qui devient la seule réponse envisageable à l'incohérence temporelle des décisions.

Aujourd'hui, la nécessité d'accompagner le cadre institutionnel par des modifications qui répondent aux contraintes des réformes est un développement positif par rapport à la vision NEC. Lorsque les marchés sont défaillants, la libéralisation financière peut ne pas contribuer positivement à la croissance (Stiglitz, 1989), ce qui débouche dans le paradigme NK sur la proposition de politiques de discrétion contrainte (Bernanke et Mishkin, 1997). Il est considéré que l'objectif d'inflation doit être pris non comme une règle rigide de comportement mais comme un cadre général d'action de façon à améliorer la communication, la transparence et la discipline de la politique monétaire à l'égard des marchés. Il est aussi suggéré qu'en raison des faiblesses des économies émergentes, la libéralisation ne doit pas être appliquée d'un seul coup mais doit être séquentielle en fonction des capacités d'absorption des structures locales (Stiglitz, 1999). Signalons que ce consensus n'est pas nouveau. Krueger (1984) précise que la libéralisation doit débuter par l'ouverture commerciale avant de s'étendre -à condition d'avoir réussi- à l'ouverture du compte du capital tandis qu'Edwards et Cox-Edwards (1987) mettent l'accent

10 Afin de ne pas alourdir l'analyse, on ne considérera pas les différentes critiques faites à l'égard de la notion d'anticipations rationnelles. Toutefois, une critique pertinente de cette notion eu égard à la conception des politiques économiques optimales peut être trouvée chez F. Hahn (1984).

11 Je dois cette remarque à Jaime Marques Pereira.

78 Economie et Institutions - n 10 et $11-1$ er \& 2e semestre 2007 
sur la crédibilité des politiques dans le processus de libéralisation engagé. Bien que pertinent, cet assouplissement de la règle rigide du modèle NEC par les nouveaux Keynésiens ne suffit pas à appréhender tout le problème de la stabilité du système monétaire qui devrait être pensé en termes de la compatibilité des réformes avec la nécessaire gestion collectivement pertinente de la confiance en la monnaie. Ce problème renvoie à la question de l'établissement du consensus social sur les politiques monétaires à suivre, ce qui nécessite une adhésion collective à une règle monétaire (par exemple, les restrictions à la croissance et au plein emploi découlant des choix de politique conservatrice).

De ce point de vue, on remarquera que la stabilisation et la libéralisation sont deux objectifs distincts qui ne sont pas forcément compatibles. La stabilisation vise à rétablir la confiance à la fois en la monnaie nationale et en le régime monétaire (politique monétaire, politique de change et financement de la dette publique), mais aussi en le régime économique de reproduction (financement de l'économie, croissance, amélioration du niveau de vie, investissements directs étrangers, IDE), ce qui nécessite un consensus d'abord national et, ensuite, international. La libéralisation est, pour sa part, une vision particulière sur le fonctionnement des marchés et impose des règles de politique économique dont la portée pour la stabilité est relative. A travers le développement de la financiarisation par la libéralisation, les régimes de change à crémaillère ou par ancrage cherchent à établir la crédibilité des politiques monétaires et la légitimité du pouvoir national aux yeux des marchés internationaux. La crédibilité financière apparaît bien comme un problème de légitimité (comme l'adhésion de l'opinion financière) dès lors que l'enjeu est l'ancrage nominal des créances sur des références monétaires extérieures. Elle est aussi un problème de légitimité politique étant donné le gonflement de la dette publique et la limite à la création de monnaie de crédit qui en découle. Mais le régime choisi peut engendrer une appréciation de la monnaie nationale qui réduit la compétitivité et concourt à la détérioration du solde commercial, pouvant déboucher paradoxalement sur l'effondrement de la crédibilité externe du système monétaire. Les déséquilibres interne et externe entrent alors en conflit avec les réformes car la résorption du déficit courant devient tributaire des flux de capitaux à court terme qui sont très sensibles aux retournements de conjoncture internationale ${ }^{12}$. Par conséquent, lorsque l'on suit une approche qui considère l'économie de marché comme une économie monétaire dont la validité des règles constitutives déterminent les conditions de viabilité du système

${ }^{12}$ Mais aussi aux événements qui peuvent être interprétés en termes des effets des tâches solaires sur les fluctuations économiques comme le soulignent Marques-Pereira et Théret (2004).

Economie et Institutions $-\mathrm{n}^{\circ} 10$ et $11-1$ er $\& 2$ e semestre 2007 
économique, les crises qui surviennent apparaissent comme la manifestation de la perte de confiance en la légitimité des choix monétaires tant sur le plan de leur application par les intervenants de marché que sur le plan de leur surveillance et de leur défense par les autorités.

La leçon de l'expérience turque est qu'en l'absence d'une légitimité monétaire établie, le besoin de financement externe, généré par le déficit commercial et les rapatriements des profits, devient insoutenable sans une réduction de la demande intérieure. Face à la menace financière, l'orthodoxie paraît la seule réponse possible alors que l'accroissement des fragilités financières et la réduction de la capacité de financement externe après un retournement de la conjoncture nationale et/ou internationale ne sont pas exempts d'effets inverses.

\section{Légitimité des règles et viabilité du régime monétaire : une interprétation de l'expérience turque}

Le rétablissement de la confiance en le régime monétaire en vigueur est nécessaire pour la stabilité du système monétaire. Dans le cas des économies émergentes comme la Turquie, la réalisation d'un tel objectif est souvent liée à l'obtention d'une légitimité internationale par une politique monétaire conservatrice et à la mise en place d'un régime financier tributaire du renouvellement à court terme de la dette croissante. Lorsque ces conditions ne sont pas remplies, les marchés financiers préfèrent, en dépit des politiques dites crédibles, ne pas répondre aux besoins croissants de liquidité de l'économie. L'économie toute entière se trouve alors placée dans une position d'insolvabilité alors que les agents économiques cherchent à se retirer de la communauté des paiements afin de sauvegarder leurs avoirs, ce qui invalide le régime monétaire en vigueur. Toutefois, la seule légitimité internationale à travers les politiques d'intégration financière ne permet de répondre ni aux faiblesses structurelles de l'économie turque ni aux problèmes de répartition et de chômage, ce qui se traduit par la fragilité persistante de la confiance interne en la monnaie nationale.

80 Economie et Institutions $-\mathrm{n}^{\circ} 10$ et $11-1$ er $\& 2$ e semestre 2007 


\section{1. Des réformes aux crises ${ }^{13}$}

A partir du début 1980 , le système bancaire et financier turc fait l'objet d'une réforme large visant à libéraliser l'ensemble des marchés dans l'objectif d'une intégration financière. Le régime monétaire mis en place s'inscrit dans la vision de crédibilité restreinte. Le programme libéral, accompagnant les événements internationaux (évolutions en Iran, en Afghanistan et au MoyenOrient), trouve un appui externe mais ne permet pas de restaurer la confiance du public en le nouveau régime et ravive le conflit distributif et politique dont la généralisation à l'ensemble de la société est évitée par le coup d'Etat militaire de septembre 1980. Ce dernier n'empêche pas qu'une première crise interne de confiance méthodique survienne mi 1982. La pression sur les taux d'intérêt et la concurrence accrue entre les banques dans la collecte des dépôts débouche sur une faillite généralisée des bancaires ${ }^{14}$ et sur la fermeture de cinq banques privées. Ces faillites, provoquant des pertes pour les déposants, entachent profondément leur confiance éthique en les institutions financières. Parallèlement, l'efficacité réduite des décisions du pouvoir en place mine la confiance hiérarchique du public en l'autorité monétaire qui, au nom de la libéralisation des marchés financiers, n'a pas su réguler les activités d'intermédiation des établissements. L'inflation durable et l'affaiblissement de la confiance éthique en la monnaie nationale réduisent l'horizon de décision des agents et les poussent vers des refuges monétaires externes. Le taux d'intérêt est utilisé par la banque centrale contre la substitution monétaire et les attaques spéculatives. Mais avec la persistance des déséquilibres et les incertitudes politiques, l'autorité se retrouve incapable de rendre opérationnelles les stratégies envisagées pour sa légitimité interne et externe : maintenir une confiance en la monnaie nationale, rétablir l'équilibre interne par une baisse de l'inflation et forger une légitimité externe en attirant suffisamment de fonds pour renouveler une dette croissante. Cette période se révèle intéressante dans la mesure où à

13 Il ne s'agit pas ici de présenter les réformes de libéralisation et d'ouverture depuis les années 1980, mais d'interpréter les crises survenues en utilisant la grille de lecture adoptée dans la première section. Pour une étude sur la fragilité du système bancaire turc et sur le rôle du financement monétaire des déficits publics croissants dans l'évolution du système dans les années 1980-90, nous nous permettons de renvoyer le lecteur à Ülgen et al. (2003).

14 Les bancaires sont les premières institutions financières dont la propriété et la direction appartenaient en général à un nombre réduit de personnes qui proposaient des taux d'intérêt beaucoup plus élevés que les banques traditionnelles en vue de collecter notamment les primes de retraite des retraités -une sorte de fonds de pension particulier- et qui réallouaient ces fonds auprès des banques ou entreprises en difficulté de liquidité.

Economie et Institutions $-\mathrm{n}^{\circ} 10$ et $11-1$ er $\& 2$ e semestre 2007 
peu près les mêmes dynamiques de crise sont à l'œuvre vingt ans plus tard.

A partir de $1988^{15}$, tandis que les réformes n'arrivent pas à créer un marché financier diversifié et profond (Atiyas et Ersel, 1994), la croissance s'essouffle et le programme anti-inflationniste bute sur des rigidités (Calvo et Végh, 1999). L'ouverture du compte du capital en 1989 produit deux effets : un accroissement de l'endettement extérieur des entreprises et une entrée nette relative de capitaux contribuant à faire pression sur les taux d'intérêt et à l'appréciation de la livre turque. L'abolition de certaines réglementations sur les produits d'importation provoque une hausse des importations débouchant sur un déficit commercial de 9,5 milliards de dollars américains en 199016. L'avis négatif de la Commission européenne sur la demande d'adhésion (fin 1989) et le conflit du Golfe (1990) détériorent la conjoncture extérieure. Le compte courant redevient déficitaire et l'inflation reprend son rythme de croisière. L'économie subit une contraction ${ }^{17}$ et le déséquilibre externe continue de se renforcer ${ }^{18}$ tandis que le besoin de financement du secteur public augmente fortement atteignant $7,4 \%$ du PNB en 1990 et $12 \%$ en 1993. Le choix des autorités de financer le déficit sur le marché intérieur débouche sur une série de mesures et les banques sont obligées de constituer $12 \%$ de leur passif en titres publics auprès de la banque centrale. L'accroissement du déficit fait pression sur les prix et les taux d'intérêt ${ }^{19}$ et imprègne les stratégies des banques dont la position ouverte s'élève à 4,98 milliards de dollars en 1993, équivalant à $178,4 \%$ du capital payé du système. La détérioration des contraintes externes et internes déclenche une forte dépréciation, une hausse des taux d'inflation et d'intérêt et débouche sur une crise réduisant la croissance de $6,1 \%$.

En effet, depuis le début de la libéralisation, le régime monétaire est fondé sur la redistribution du revenu national en

15 Pour une présentation des réformes du système bancaire turc dans les années 1980-90, nous nous permettons de renvoyer le lecteur à Ülgen (2007).

16 Sauf indication du contraire, toutes les données sont obtenues à partir des statistiques disponibles sur les sites de la Banque Centrale de la République Turque (tcbm.gov.tr), du Trésor (hazine.gov.tr) et de l'Institut de statistiques turc (tuik.gov.tr).

${ }^{17}$ Le taux de croissance du PNB passe de 9,8\% en 1987 à 1,6\% en 1989 .

18Le solde du compte courant en 1990:-2,625 milliards de dollars, en 1991 : -6,433 milliards (balance commerciale : -14,08 milliards). Le taux de couverture baisse de 81,4\% en 1988 à 52,1\% en 1993 .

${ }_{19}$ L'indice des prix à la consommation (IPC) en moyenne annuelle passe de $37 \%$ en 1986 à $71,7 \%$ en 1988 pour s'établir à 68\% en 1993 alors que le taux d'intérêt sur les dépôts à un an évolue, respectivement, de $48 \%$ à $83,9 \%$ et à $74,7 \%$.

82 Economie et Institutions - n 10 et $11-1$ er \& 2e semestre 2007 
faveur des créditeurs et sur les besoins d'endettement croissants (Akyüz, 1992). Son horizon de viabilité se réduit à des périodes comprises entre deux grandes difficultés systémiques (1980-1982, 1983-1988, 1989-1994, 1995-1999, 1999-2001) et est établi sur une confiance à court terme des flux spéculatifs. Mais comme la confiance méthodique n'est pas renforcée, les comportements des agents, constamment aux aguets, se transforment fréquemment en une crise interne générale qui, dédoublée d'une crise externe, augmente en puissance à deux reprises.

En avril 1994, le gouvernement annonce un ensemble de mesures qui ne modifient pas la structure des difficultés antérieures. En dépit de l'objectif de développement des marchés financiers à travers les opérations privées de titrisation et de diversification des produits financiers, annoncé au début des années 1990, la part des titres privés dans le stock des actifs financiers descend en dessous de $15 \%$ au profit des titres publics (Ülgen et al. 2003, pp. 5-6). Sous le contrôle du FMI, le gouvernement débute à partir de mi-1998 un nouveau programme de désinflation. La crise russe d'août 1998, les élections législatives d'avril 1999 et deux grands tremblements de terre vers la fin de 1999 engendrent des pressions sur les comptes publics. Le ralentissement de la croissance et la persistance d'une inflation forte $(68,2 \%)$ appellent un autre programme à la fin de 1999 qui vise à réduire l'inflation structurelle à partir de la stabilisation du taux de change ${ }^{20}$ et de la dette extérieure. Toutefois, les marges de manœuvre dont la banque centrale dispose pour rendre cohérents à la fois l'objectif de stabilisation des prix et le déséquilibre courant se révèlent très réduites. La faiblesse des moyens et des modalités de financement du déficit et de l'activité économique sur des ressources longues, empêchant la confiance méthodique de se rétablir, constitue une raison remarquable de rupture.

La viabilité du régime dépend aussi de la stabilité du système bancaire mais les banques s'attachent à réaliser des profits dans le financement de la dette publique ${ }^{21}$. Suivant l'ancrage défendu par l'autorité, les positions ouvertes ${ }^{22}$ continuent de s'accumuler rendant la solvabilité bancaire tributaire de la stabilité du change à l'instar de certaines expériences en Amérique latine, étudiées par Velasco

2011 s'agit d'un régime de change à crémaillère, fondé sur un ancrage (la livre turque est calquée sur un panier constitué de 1 \$US et de $0,77 €)$ devant compenser d'éventuels différentiels d'inflation en vue de réduire les effets sur la compétitivité.

${ }^{21} \mathrm{La}$ part de la dette sécurisée du gouvernement central détenue par les banques commerciales suit ainsi une trajectoire ascendante : 1995 : 20,3\% ; 1996 : $31,1 \%$; 1997 : $54,6 \% ; 1998$ : 59\% ; 1999 : 63\% ; 2000 : 67,3\% ; 2001 : 53,4\% (Source : FMI).

${ }^{22} \mathrm{La}$ position ouverte des banques commerciales croît d'une façon remarquable : 4,6 milliards de dollars en 1999 et 8,55 milliards en 2000 .

Economie et Institutions $-\mathrm{n}^{\circ} 10$ et $11-1$ er $\& 2$ e semestre 2007 
(1987). L'incitation à renforcer les bilans bancaires est réduite (Eichengreen, 2001) alors que le secteur n'arrive pas à asseoir son efficacité (Denizer et al. 2000) et que la qualité de ses engagements se dégrade au profit des opérations à haut risque le rendant activement responsable de la détérioration de la stabilité monétaire et financière (Miotti et Plihon, 2001). En effet, s'appuyant sur une confiance hiérarchique spéculative, les banques marchent du même pas à la recherche de gains élevés dans le financement du déficit public et sans se préoccuper de la fragilité de leur position courte cumulée. Or, la capacité de l'économie à faire recycler sa dette s'affaiblit si bien que les accords de la "marche turque " ne semblent plus assez harmonieux pour que les créanciers aussi acceptent de continuer à marcher du même pas que les banques nationales.

Alors que la politique de développement est fondée, depuis les années quatre-vingt, sur les flux de capitaux encouragés par les différentiels de taux d'intérêt, le service de la dette (qui passe de 11,4 milliards de dollars en 1996 à 22 milliards en 2000 dont, respectivement, 4,2 et 7,134 milliards pour les seuls paiements d'intérêt) joue en défaveur de la garantie fournie par les autorités en vue de réduire la méfiance sur la capacité de l'économie à répondre à la contrainte extérieure et à assurer la viabilité du régime dans le temps. Ainsi, l'année 2000 évolue sur le fil du rasoir. Les problèmes de légitimité politique et les fragilités économiques et financières dominent les comportements attentistes des agents. 5 banques privées insolvables sont transférées au Fonds d'Assurance des Dépôts. Demirbank, qui avait accumulé des titres publics à travers ses emprunts courts, rencontre des problèmes de liquidité qui l'obligent à procéder à une vente massive de titres en novembre 2000. La faiblesse de la confiance méthodique apparaît alors déterminante et les taux d'intérêt accusent de très fortes hausses et les anticipations des marchés se tournent vers une dévaluation proche $^{23}$. Le degré de liquidité de l'économie se réduit fortement, les flux de capitaux se renversent et la crise externe de confiance méthodique devient réelle ${ }^{24}$. Malgré une ligne supplémentaire de

23Fin novembre, le plafond des taux courts (1 jour - 1 mois) passe de $70 \%$ à $300 \%$ et le 4 décembre, il atteignait $2500 \%$. La banque centrale a voulu rester sur sa politique restrictive afin de conserver la crédibilité du programme alors que le solde de la balance commerciale accusait un déficit élevé (qui était descendu de $-14,22$ milliards de dollars à $-10,44$ milliards entre 1998 et 1999, est remonté à -22,37 milliards en 2000). Le solde du compte courant passait de 1,98 milliard de dollars en 1998 à -1,36 milliard en 1999 et à $-9,819$ milliards en 2000. Le ratio du solde courant aux réserves nettes de change de la banque centrale, qui était de 9,57\% en 1998 , était de $-5,56 \%$ en 1999 et de $-42,41 \%$ en 2000 .

${ }^{24}$ Pour 1998 et 2000, les investissements directs étrangers (IDE) étaient de 0,573 et 0,112 milliard de dollars alors que les investissements nets de

84 Economie et Institutions - n 10 et $11-1$ er \& 2e semestre 2007 
crédit de 7,5 milliards de dollars par le FMI en décembre 2000 et la garantie offerte par le gouvernement sur les avoirs étrangers, la confiance hiérarchique ne suffit plus à enrayer la méfiance méthodique et éthique et une crise profonde survient en février 200125. La confiance méthodique des créanciers est ébranlée puisque l'autorité hiérarchique ne semble être en mesure d'ordonner ni les comportements des banques ni la stabilité de la monnaie nationale. La spéculation contre la monnaie nationale l'emporte et les règles monétaires nationales se disloquent sous l'effet d'une double crise, interne et externe. Le risque de système apparaît alors à deux niveaux : perte de signification pour les règles nationales (apparition des références monétaires allogènes ${ }^{26}$ ) et recherche de la préservation des intérêts privés provoquant la ruée.

\section{2. Effritement des confiances}

La survenance et la sortie d'une crise posent d'une façon récurrente la question de la pertinence structurelle des réformes et leur portée systémique étant donnés les coûts de plus en plus exorbitants des essais in vitro (Carstens et al. 2004). Alors que l'on met l'accent habituellement sur l'infrastructure institutionnelle, comme l'indépendance de la banque centrale ou le développement du système financier, il conviendrait de remarquer que la stabilité monétaire dépend avant tout du choix des réformes puisque celles-ci prédéterminent la capacité du système à se rétablir d'une façon pertinente dans le temps.

L'inflation persistante dans les années 1980-90, sans avoir débouché sur une hyperinflation, semble révéler les difficultés d'aboutir en Turquie à un état institutionnalisé de la monnaie autour des règles cohérentes et effectives. La règle de l'ancrage du change permet de diffuser dans la société les conditions éthiques que requiert la stabilité des prix mais n'opère qu'une restauration illusoire des trois formes de la confiance. Les dettes croissantes et l'endettement public excessif impliquent tôt ou tard une crise interne du système de monnayage, témoignant de l'erreur de conception de

portefeuille s'établissaient à, respectivement, $-6,711 ; 1,022$ milliards. En novembre 2000, il y a eu une sortie de 5,037 milliards. Les cinq premiers mois de l'année 2001, la sortie nette était de 3,562 milliards.

${ }^{25}$ Une dépréciation de près de $60 \%$, les taux courts ont fluctué jusqu'à $5000 \%$ entre le $21 / 12 / 2000$ et le $19 / 03 / 2001$ pour descendre à $66 \%$ fin 2001.

${ }^{26} \mathrm{La}$ part des actifs libellés en devises étrangères sur le total des actifs est passée de $26 \%$ en 1998 à 38\% en 1999 et celle des passifs libellés en devises sur le total des passifs de $25 \%$ à $48 \%$. La part des dépôts bancaires en devises sur le total des dépôts était de 49,9\% en 1999 et de 60,97\% en 2001 avec une échéance moyenne de 3 mois.

Economie et Institutions $-\mathrm{n}^{\circ} 10$ et $11-1$ er $\& 2$ e semestre 2007 
la recentralisation de la communauté de paiements à partir des seules conditions éthiques de la stabilité des prix. En Turquie, cette crise interne explose sous forme de crise externe qui voit s'effondrer tant la souveraineté monétaire que la souveraineté politique.

En reprenant la grille de lecture proposée dans la première partie de ce travail, il est possible de montrer l'état des trois formes de confiance dans l'économie turque. Pour ce faire, nous identifions les comportements des différentes catégories d'agents et leurs réactions aux évolutions économiques et financières. Les comportements et réactions des deux premières catégories, les ménages/salariés et le système bancaire national, par rapport au régime monétaire en vigueur, déterminent l'intervalle de viabilité ou la survenue de la crise interne tandis que ceux de la troisième catégorie, que nous appelons investisseurs étrangers, définissent le seuil de la crise externe.

En ce qui concerne les ménages/salariés, les réactions aux conséquences des politiques de rigueur se transforment en une défiance vis-à-vis du régime monétaire. La confiance dans l'autorité monétaire est réduite, autorité qui est vue comme un intermédiaire peu scrupuleux entre les financeurs des programmes (le FMI) et les rentiers sans que les politiques mises en œuvre soient perçues comme capables d'améliorer le niveau de vie du public. La confiance méthodique est aussi ébranlée en fonction du conflit distributif et des attentes inflationnistes. L'absence d'un ordre routinier, nécessaire pour considérer l'avenir avec sérénité, empêche les ménages de recouvrer leur confiance en le régime monétaire, modifié dans sa composante 'gestion du change' mais reconduit dans sa composante 'conservatrice'. L'évolution défavorable des salaires et les dépenses publiques inefficaces (détérioration des infrastructures sociales, éducatives) renforcent la méfiance. La fuite vers des monnaies allogènes (la substitution monétaire : dépôts bancaires en devises sur l'ensemble des dépôts est de 50-60\% sur 1994-2002) et le raccourcissement des échéances des dépôts en découlent comme des réactions remarquables. La confiance éthique est également entamée avec le détournement des épargnes et l'épuisement du pouvoir d'achat. Les crises se soldent en général par un transfert des richesses vers des centres financiers extérieurs et réduit fortement la richesse nationale. L'autorité hiérarchique n'est pas considérée comme étant capable de faire respecter aux différentes parties les règles éthiques du régime monétaire et d'assurer les conditions d'une croissance pérenne.

Quant au système bancaire national, la confiance des banques en le régime monétaire est très spéculative et instable dans la mesure où à la moindre secousse les établissements n'hésitent pas à procéder à des ventes massives de leurs détentions provoquant ou aggravant une crise de liquidité autoréalisée. Le financement des

86 Economie et Institutions - n ${ }^{\circ} 10$ et $11-1$ er \& 2e semestre 2007 
positions ouvertes des banques nationales est néanmoins assuré sur la base des confiances hiérarchique et éthique, fondées sur l'engagement des autorités et sur l'intervention des bailleurs de fonds en dernier ressort (le FMI). La confiance hiérarchique est établie à court/moyen termes sur la politique monétaire et le régime de change défendus par les autorités. Le financement du déficit à travers le système bancaire à des taux d'intérêt élevés fait apparaître une rente sur la dette publique sécurisée. La part de la dette du gouvernement central détenue par les banques commerciales passe de $20,3 \%$ en 1995 à $67,3 \%$ en 2000 des actifs bancaires. Mais cette confiance est spéculative puisque les banques savent qu'à court terme, la situation macroéconomique ne sera pas rapidement assainie. La confiance méthodique est faible ; les anticipations sur le renouvellement des opérations d'endettement du système productif dans des conditions adéquates sont ébranlées par l'incapacité de l'économie à devenir compétitive. Il s'ensuit une faiblesse inquiétante des activités bancaires traditionnelles par rapport aux pays comparables ; le ratio du Crédit bancaire/PNB s'établit à 21,72\% sur 1994-1998; en 2002, il est de 13,32\%. Le ratio du Crédit à l'économie/Actifs bancaires du secteur privé est de 30\% et celui du crédit bancaire au secteur privé de 20\% du PNB sur 1998-2003. La remonétarisation de l'économie n'est pas rétablie et l'échéance des passifs bancaires reste courte (pour les dépôts en devises, elle est, en moyenne, de 2,7 mois et pour les dépôts en livre turque de 3 mois). La confiance éthique est sur le fil du rasoir car la structure du régime monétaire en vigueur est caractérisée à terme par un accroissement des fragilités et chaque établissement cherche à profiter au maximum des opportunités spéculatives fournies par cette évolution instable qui implique tôt ou tard une liquidation des bilans des établissements ayant des positions ouvertes insolvables (courtes).

On remarque que la méfiance du public (ménages/salariés) vis-à-vis des trois niveaux de confiance entre en conflit permanent avec la confiance "formelle" des banques vis-à-vis du régime. Néanmoins, la confiance du système bancaire l'emporte sur la méfiance du public et permet d'orienter les décisions des déposants vers la fuite devant l'accroissement des faiblesses du système productif et du système bancaire. Cette fuite, hormis le phénomène de substitution monétaire, s'effectue aussi sous la forme de l'accroissement des dépôts bancaires à court terme à fort taux d'intérêt, ce qui traduit une sorte d'acceptation par le public de continuer à alimenter la liquidité bancaire à très court terme. Cette résignation de la méfiance devant les promesses sans fondement d'un rendement immédiat des engagements est un trait caractéristique de la vision spéculative court-termiste. Faute d'un 
horizon éloigné, beaucoup de déposants deviennent inconsciemment risk lovers.

Concernant la troisième catégorie d'agents, les investisseurs étrangers dont les comportements traduisent l'état de la confiance externe, leurs décisions sont dominées par une méfiance sur l'évolution de la dette publique, du déficit courant et sur la capacité compétitive de l'économie, ce qui se traduit par des taux d'intérêt élevés et une grande volatilité des flux de capitaux. La méfiance sur la viabilité du régime est très présente, mais cette fragilité permet aux flux de se transformer volontiers en des entrées de fonds chauds alimentant la volatilité des déséquilibres et de soumettre l'économie à leur sensibilité exubérante. La confiance hiérarchique en l'autorité est établie sur les politiques d'ouverture et monétaires de rigueur (selon les règles $\mathrm{NEC} / \mathrm{NK}$ ), mais dépend surtout de la garantie publique des créances et des évolutions conjoncturelles (négociations avec l'Union européenne ou programmes soutenus par le FMI). Bien que cette confiance évolue en fonction de la stabilité du change et donc de la règle de convertibilité externe de la monnaie nationale en parallèle avec l'évolution de la dette publique, elle est principalement fondée sur une vision spéculative et ne se transforme pas en une confiance méthodique puisque l'adhésion de l'opinion financière à la légitimité du régime est à court terme (rentabiliser une situation dégradée de la dette) et attentiste à l'égard de l'inflation.

Les attraits spéculatifs de l'économie permettent de satisfaire la confiance fragile des investisseurs à court terme. Mais comme les confiances ne sont pas véritablement restaurées d'une manière continue, les flux de capitaux n'accompagnent pas automatiquement les essais de rétablissement des confiances internes à travers l'assainissement du système bancaire et la baisse de l'inflation, observés depuis la crise de 2000-2001. L'adoption de la politique monétaire anti-inflationniste permet à court terme de retrouver une plus grande stabilité des prix et de continuer les réformes structurelles. Mais cette séquence évolue dans un déficit de légitimité sous-jacent au régime de reproduction posant la question de savoir si le régime monétaire réformé peut rendre soutenable la dette publique et faire en sorte que les gains financiers soient compatibles avec une relance également soutenable de l'accumulation du capital. Ceci exige le maintien de l'élasticité négative des salaires au besoin de financement externe qui ne cesse d'augmenter, dû en partie au déficit croissant du compte courant. La situation actuelle de l'économie turque traduit précisément l'ambiguïté des effets positifs observés des modifications du régime monétaire depuis que les autorités ont adopté en 2001 une politique de règle d'inflation avec un régime de flottement des changes.

88 Economie et Institutions - n ${ }^{\circ} 10$ et $11-1$ er \& 2e semestre 2007 


\subsection{La stabilité macroéconomique dans l'après-crise et la portée du régime monétaire réformé}

Il est certain que la crise de 2000-2001 a provoqué un changement de conception dans le choix des politiques monétaires, qui se révèlent plus attentives à l'évolution du déficit public et du niveau des prix; la banque centrale est devenue officiellement indépendante depuis avril 2001.

De nombreux indicateurs dénotent une amélioration relative des fondamentaux concernant la stabilité macroéconomique et le rétablissement de la confiance externe (à travers les réactions des capitaux étrangers et des organismes de refinancement comme le FMI) et interne. Ainsi, entre 2003 et juin 2006, le stock net de la dette extérieure rapporté au PNB passe de $21,9 \%$ à $7,1 \%$, le stock de la dette publique totale de $70,3 \%$ à $50,1 \%$ et celui de la dette interne de $48,4 \%$ à $43 \%$.

Toutefois, malgré un excédent primaire qui passe de $-1,9 \%$ du PNB en 1999 à $6,2 \%$ en 2003, le service de la dette extérieure (remboursement du principal et des charges d'intérêt) constitue un poids important sur l'équilibre financier de l'économie. De 27,810 milliards de dollars en 2003 (dont 6,987 milliards pour les intérêts) il passe à 39,751 milliards en 2006 (dont 9,346 milliards pour les intérêts). La dette extérieure totale, qui était de 144,301 milliards en 2003 (dont 23,013 milliards à court terme et 121,288 milliards à long terme), s'établit à 207,746 milliards en 2006 (avec 42,326 milliards à court terme et 165,42 milliards à long terme) et à plus de 237 milliards à la fin du troisième trimestre 2007, traduisant une constante augmentation.

Pour sa part, le taux d'intérêt réel sur la dette intérieure passe de $11,91 \%$ en 2003 à $7,79 \%$ en 2006 pour remonter à $9,78 \%$ en $2007^{27}$. De même, le taux moyen annuel des adjudications des Bons du Trésor descend de 46\% en 2003 à 16,3\% en 2005 pour remonter à $18,42 \%$ en 2007 , ce qui se situe parmi les taux les plus élevés au monde.

La composition de l'échéance de la dette intérieure traduit un allongement de la durée des émissions publiques et la part des obligations d'Etat par rapport aux émissions des Bons du Trésor s'accroît. Ainsi, de 55,3\% des émissions avec une échéance moyenne de 20 mois en 2003 (contre 35,2\% pour les Bons du Trésor avec une échéance moyenne de 6,2 mois), les Obligations d'Etat voient leur part augmenter à $84,7 \%$ avec une échéance de 38,9 mois en 2007 (14,4\% pour les Bons du Trésor avec une échéance de 5,1 mois), témoignant d'un relâchement de la contrainte temporelle.

27 La moyenne de juin à septembre 2007.

Economie et Institutions $-\mathrm{n}^{\circ} 10$ et $11-1$ er $\& 2$ e semestre 2007 
Cependant, malgré cette relative amélioration, il convient de souligner que cette évolution traduit l'importance persistante de la dette publique dans les émissions intérieures. Ceci reste une caractéristique structurelle de l'économie et renvoie aux difficultés de développer les marchés financiers (en termes d'approfondissement et d'élargissement) dans l'objectif d'une plus grande résilience de la sphère financière aux chocs extérieurs et intérieurs. En effet, dans le portefeuille de titres détenus par le système bancaire, la part des Obligations d'Etat, qui était de $55 \%$ en 2003, suit toujours une évolution ascendante passant à $63 \%$ en 2005 et à environ $85 \%$ en octobre 2007, accompagnée de celle des Eurobonds émis par le Trésor (qui s'établit, respectivement, à $8,3 \% ; 5,8 \%$ et à $11,4 \%$ pour les mêmes dates) tandis que la part des titres privés (hors titres mis en nantissement) s'établit à $2,3 \%, 1,8 \%$ et à $2,5 \%$ pour les mêmes années.

Ainsi, même après une période de forte consolidation, la profitabilité du secteur bancaire dépend principalement des rendements élevés des titres publics et la contribution des activités bancaires traditionnelles reste toujours très faible. Selon le Rapport de la Banque Mondiale (WB, 2006), le ratio du Crédit bancaire au secteur privé/PNB de l'économie turque se situe parmi les plus faibles des économies émergentes avec l'Argentine et le Mexique. Pour l'ensemble des banques privées, la variation du volume des crédits commerciaux, qui était de $36,5 \%$ en $2003(-2,9 \%$ en 2002$)$ et de $44,2 \%$ en 2005 , était de $18,9 \%$ en 2006 et de $16,6 \%$ en 2007. Pour l'ensemble des banques, la part des crédits commerciaux reste en dessous de $20 \%$ en 2007.

On observe toutefois une amélioration dans l'évolution des parts des actifs et des passifs en devises étrangères dans le bilan du système bancaire. La part des actifs en devises du système passe ainsi de $38 \%$ en 2003 à 33, $1 \%$ en 2006 et à $28,9 \%$ en octobre 2007 tandis que celle des passifs en devises se situait, respectivement, à $43,3 \%, 37,8 \%$ et à $34,5 \%$, ce qui peut être expliqué en partie par le changement de politique du système bancaire qui consiste à rémunérer les dépôts ordinaires en devises à des taux parfois inférieurs aux taux appliqués sur les dépôts à terme dans les pays européens. Mais cette situation reste dépendante de facteurs externes au système bancaire : l'évolution descendante des taux d'intérêt internationaux et la stabilité du taux de change sur cette période. Un autre facteur significatif est le différentiel d'intérêt favorable au marché financier national qui constitue la condition d'attractivité des placements en livre turque mais défavorise le coût de financement interne des investissements productifs.

Une autre évolution positive mais sujette à caution est le processus de désinflation devenu significatif après la crise ; de $68,5 \%$ en 2001 , le taux d'inflation est passé à $18,4 \%$ en 2003 et à $7,7 \%$ en

90 Economie et Institutions - n ${ }^{\circ} 10$ et $11-1$ er $\& 2$ e semestre 2007 
2005. Mais il se révèle que le passage à un régime de change flexible ne résout pas le problème des déséquilibres interne et externe. D'une part, les autorités ne sont pas en mesure de contenir l'inflation en cas d'une dépréciation étant donnée la dépendance financière et monétaire de l'économie vis-à-vis de l'extérieur, ce qui a été observé dans la reprise de l'inflation à partir des retournements dans la conjoncture internationale au début de l'été 2006 (9,6\% en 2006 et $8,4 \%$ en 2007 , dépassant de loin l'objectif annoncé de $4 \%$ pour ces mêmes années). D’autre part, la compétitivité de l'économie ne semble pas s'améliorer si bien que le déficit courant continue de faire pression sur les taux d'intérêt nationaux en même temps qu'un grand nombre de fragilités qui minent la confiance des agents en le régime monétaire en vigueur persistent. Malgré de forts taux de croissance du PNB, passant de $-9,5 \%$ en 2001 à $7,9 \%$ en 2002 , à $9,9 \%$ en 2004 et à $7,6 \%$ en 2005, l'évolution de l'indice réel des salaires reste faible (pour le secteur manufacturier, à $90 \%$ de son niveau moyen de la période 1994-99), traduisant une absence d'amélioration du niveau de vie. Depuis 2002, les forts taux de croissance et la baisse de l'inflation ${ }^{28}$ semblent contenir la méfiance des ménages vis-à-vis du régime monétaire alors que l'évolution du taux de chômage, en partie due aux pressions démographiques ${ }^{29}$, traduit la persistance des faiblesses de l'économie turque ${ }^{30}$. Il faudrait, par conséquent, une croissance soutenue dans les secteurs non agricoles afin de résorber le chômage. Mais la dynamique économique des dernières années ne semble pas suffire à créer ces emplois (Gürsel, 2007).

Il est à remarquer aussi que depuis 2004 , les flux de capitaux ne cesse de croître pour atteindre, sur la période janvier-novembre 2007, une valeur égale à 31,093 milliards de dollars. Par rapport à la faiblesse des IDE des années antérieures (en 2003, les IDE étaient de

28 Les politiques monétaires anti-inflationnistes, bien que conservatrices, semblent constituer une valeur éthique universelle acceptée aujourd'hui même par les syndicats et les classes populaires comme critère positif d'évaluation des actions des gouvernements.

29 L'augmentation soutenue de la population active continue sous la pression de trois facteurs : la dynamique démographique (accroissement de la population en âge de travailler), la hausse du taux d'activité des femmes (effet de travailleur additionnel dû en partie à la baisse des salaires réels) et le transfert de la main-d'œuvre agricole vers d'autres secteurs sous l'effet, notamment, des rigueurs budgétaires de l'après-crise qui ont réduit les subventions agricoles (voir Gürsel, 2007).

30 Le taux de chômage (de la population active totale des 15 ans et plus), qui était de 10,5\% en 2003, ne commence à baisser que très légèrement en 2006 et 2007 s'établissant à 9,9\% en moyenne, le taux de chômage non agricole se situant à 12,6\% en 2006 et à 13\% fin 2007. Le dernier taux disponible est de $14,2 \%$ en février 2008 (11,6\% pour le taux de chômage général) tandis que le chômage des jeunes se situe aux alentours de 20\% fin 2007 selon le TUIK.

Economie et Institutions $-\mathrm{n}^{\circ} 10$ et $11-1$ er $\& 2$ e semestre 2007 
1,752 milliard et en 2004, de 2,885 milliards), qui manifestait une absence de confiance en les capacités de reproduction de l'économie, en 2005, les IDE atteignent 10,029 milliards et en 2006, près de 20 milliards. Cette évolution est en partie due à une nouvelle loi qui a davantage libéralisé le régime réglementaire sous-jacent aux IDE. Toutefois, les IDE des non-résidents en Turquie ${ }^{31}$ se sont établis à 16,691 milliards de dollars en janvier-novembre 2007, en dessous de leur niveau de 2006 et les IDE nets à seulement 14,699 milliards. Les secteurs les plus représentatifs des IDE ont été, en 2005 et 2006, celui des transports et communication et celui de l'intermédiation financière, qui sont les principaux secteurs dans lesquels les privatisations ont été suivies avec 8,22 milliards de dollars en 2005 et de 8,1 milliards en 2006 de valeur de vente. De ce point de vue, les IDE greenfield ${ }^{32}$ restent pour l'instant relativement limités, hormis les entrées de capitaux, dans les secteurs privatisés suite à la décision du gouvernement d'abolir en 2004 les restrictions concernant le rachat des entreprises nationales de télécommunication et minières par les groupes d'origine étrangère.

Dans l'ensemble, bien que la structure de l'économie évolue vers un partage des activités en faveur du secteur des services (part sectorielle dans PIB est de 59,7\% en 2005) et de celui de l'industrie $(28,9 \%$ en 2005$)$ et en dépit d'un coût unitaire moyen du travail très compétitif, de $0,26(0,55$ pour l'UE à 15 et 0,41 pour la Bulgarie en 2004), la compétitivité internationale de l'économie ne semble pas acquise et une forte dépendance vis-à-vis des flux de capitaux de court terme est à noter comme une faiblesse structurelle accompagnant la fragilité de l'évolution du déficit du compte courant. En effet, le déficit du compte courant, en détérioration depuis 2002, passe de 9,036 milliards de dollars en 2003 à 22,604 milliards en 2005 pour s'établir à 32,758 milliards pour les 11 premiers mois de 2007, avec un fort déficit commercial de plus de 42 milliards (alors qu'il était de 14,01 milliards en 2003 et de 33,530 milliards en 2005).

En ce qui concerne la confiance éthique, la garantie publique sur les engagements bancaires mais aussi sur la dette externe croissante des entreprises permet de continuer les opérations spéculatives à court terme. Toutefois, l'évolution des déséquilibres interne et externe (comme le creusement du déficit commercial) oblige les investisseurs à rester sur leur garde afin de ne pas subir les conséquences d'une dépréciation soudaine ou d'une baisse du différentiel des taux d'intérêt. Face à ces faiblesses, deux risques majeurs potentiels planent encore sur la stabilité économique et

31 qui incluent les prêts reçus des sociétés-mères à l'étranger et les achats d'immobiliers des non-résidents en Turquie.

32 Les IDE nouveaux, créateurs de capacité et d'emplois.

92 Economie et Institutions $-\mathrm{n}^{\circ} 10$ et $11-1$ er \& 2e semestre 2007 
financière et déterminent l'intervalle de viabilité du régime monétaire en vigueur :

- risques d'origine purement externe comme l'assèchement des liquidités internationales suite à la crise actuelle des prêts hypothécaires aux Etats-Unis d'Amérique et l'augmentation soudaine des taux d'intérêt internationaux qui peuvent être combinées avec une vague de fuite des capitaux des marchés émergents et

- risques politiques internes/externes comme un affaiblissement dans les engagements du gouvernement central en matière de politique économique (la persistance d'un chômage élevé et la faiblesse de la croissance économique) ou dans ses relations avec l'Union européenne (World Bank, 2006).

\section{Conclusion}

La capacité de la politique économique à prévenir les crises et à éviter la réduction de la croissance dépend de la confiance en la viabilité du système monétaire. Le rôle des institutions et de l'Etat est lié à la question de l'intégrité du système de paiements. Dans ce cadre, l'approche développée dans ce travail questionne la légitimité du régime monétaire suivi à travers le choix des politiques monétaire et de change en prenant appui sur l'expérience de l'économie turque entre 1980-2007. On souligne alors que l'analyse des crises monétaires et financières doit porter explicitement sur le problème de la gestion collectivement pertinente de la confiance en la monnaie qui reflète le degré du consensus social sous-jacent, comme condition de soutenabilité du système de paiements et des transactions économiques et financières. Cette confiance est fortement reliée à la stabilité macroéconomique qui dépend du régime de croissance et des conditions de reproduction sous-jacentes.

Les dysfonctionnements dans le système monétaire et financier correspondent au non respect des règles du système, ce qui empêche la monnaie de remplir, aux yeux des agents économiques, ses qualités essentielles : assurer les transactions et permettre le règlement des soldes. L'affaiblissement ou la disparition de ces qualités est la traduction de l'effritement de la confiance en le régime monétaire établi et peut amener à des situations telles que la monnaie nationale peut être substituée par une monnaie étrangère. La souveraineté monétaire est alors ébranlée. Cette substitution réelle ou potentielle peut empêcher l'autorité politique de contrôler et/ou d'orienter l'économie nationale, ce qui peut grever sa souveraineté politique.

Les caractéristiques de la crise turque de 2000-2001 traduisent une longue évolution, pendant plus de deux décennies, vers la faillite de l'Etat et du système bancaire, la dépression de l'activité économique et une paupérisation forte. Elles montrent que 
la stabilisation n'est pas une simple affaire de taux de change et de politique monétaire restrictive, mais d'un consensus national et international sur les conditions de coexistence économique et politique et sur le régime monétaire choisi. C'est en fonction de son aptitude à jouer un rôle d'arbitre dans la résolution des crises et dans la refondation de la structure productive que l'Etat peut rebâtir sa légitimité politique. Par conséquent, l'arbitrage sur le nouveau régime monétaire, qui établit logiquement les nouvelles conditions de fonctionnement de l'économie tant à l'intérieur que dans ses relations avec l'extérieur, détermine si la souveraineté monétaire et la stabilité macroéconomique peuvent être recouvrées ou non.

L'économie turque de l'après crise suit une évolution ambivalente. Dans les quatre dernières années, le taux de croissance moyen avoisine $7 \%$ avec un accroissement notable de l'entrée de capitaux tant sous forme d'IDE que sous forme d'investissements de portefeuille. Les échanges extérieurs augmentent d'une façon continue et traduisent une intégration très forte de l'économie dans les circuits internationaux. La résilience de l'économie par rapport aux événements politiques intérieurs semble devenir plus forte comme le montre la stabilité monétaire et financière actuelle face aux mouvements de contestation durant les dernières élections législatives et présidentielles et à la résurgence de l'instabilité politique intérieure due aux problèmes à l'Est du pays. La stabilité de la livre turque par rapport aux principales devises, observées depuis plusieurs années, contribue à la stabilité monétaire et à l'acceptation du régime monétaire par les agents économiques. Toutefois, en $2007 / 2008$, l'évolution est loin de montrer que les stabilités interne et externe sont enfin consolidées, le taux de croissance descendant aux alentours de $4 \%$, le taux d'inflation reprenant un rythme plus soutenu et l'évolution du chômage ne s'établissant pas sur un sentier décroissant. La faiblesse de la compétitivité de l'économie et le creusement du déficit courant continuent de menacer la stabilité alors que face à la pression démographique, un taux de croissance minimal de $7-8 \%$ par an est requis dans l'avenir. Or, le régime de croissance en vigueur commence à s'essouffler et la forte dépendance de la sphère productive vis-à-vis des financements externes persiste.

La coordination efficace de la gestion macroéconomique se trouve contrainte de subir l'évolution défavorable de la conjoncture internationale en matière énergétique et en ce qui concerne la volatilité des mouvements de capitaux dans les économies émergentes. Dans l'hypothèse d'une hausse des taux d'intérêt internationaux et d'une baisse des IDE, la résorption d'une dette colossale ne serait plus envisageable et la situation macroéconomique pourrait se dégrader en provoquant une crise externe. Il est fort probable qu'en cas de la continuité des détériorations tant structurelles que conjoncturelles (coût de

94 Economie et Institutions $-\mathrm{n}^{\circ} 10$ et $11-1$ er \& 2e semestre 2007 
l'énergie, fortes perturbations actuelles sur les marchés financiers internationaux, instabilités régionales -passes d'armes avec l'UE sur la question chypriote et sur le processus de négociations d'adhésion), la frilosité des capitaux augmentera et le poids de la dette interne et externe accroîtra les tensions sur les taux d'intérêt.

Mais au-delà des incertitudes macroéconomiques, peut-on considérer désormais comme établies les conditions éthiques qui garantiraient les propriétés techniques du système de prix en évitant les brises du voile monétaire sur l'économie réelle ? Les années 1990 et 2000 sont, de ce point de vue, une période d'apprentissage de la connaissance du modèle "vrai " de l'économie, qui s'est avéré chaotique. La stabilité monétaire que permettent les politiques monétaire et budgétaire conservatrices et la concurrence peut se révéler illusoire. La seule crédibilité calquée sur une politique monétaire restrictive, même discrétionnaire contrainte, ne semble pas suffisante pour réformer et stabiliser une économie et un système monétaire hautement fragiles. Il faut un temps long et des conditions capricieuses pour rétablir la confiance des agents en le régime monétaire alors que la survenance de la crise peut se contenter de peu de nouvelles alarmantes.

\section{Références bibliographiques}

Aglietta, M. et A. Orléan (éd.), (1998), La monnaie souveraine, Paris, Editions Odile Jacob.

Aglietta, M. et al. (1998), "Introduction", dans M. Aglietta et A. Orléan (éd.), Paris, Editions Odile Jacob, pp. 9-31.

Aglietta, M. et J. Cartelier, (1998), "Ordre monétaire des économies de marché ", dans M. Aglietta et A. Orléan (éd.), Paris, Editions Odile Jacob pp. 129-157.

Aglietta, M. et A. Orléan (2004), La monnaie entre violence et confiance, Paris, Editions Odile Jacob.

Akyüz, Y. (1992), "On financial deepening and efficiency", UNCTAD Discussion Paper, $\mathrm{N}^{\circ} 43$, Geneva.

Arestis, P., M. Nissanke and H. Stein, (2003), "Finance and Development: Institutional and Policy Alternatives to Financial Liberalization", The Levy Economics Institute WP 377.

Atiyas, I. and H. Ersel, (1994), "The impact of financial reform: The Turkish experience ", in G. Caprio, I. Atiyas and J. H. Hanson (eds.), Financial reform. Theory and experience, Cambridge University Press, pp. 103-139.

Bagehot, W. (1978) (1873), Lombard Street, The Collected Works of Walter Bagehot, vol.9, London, The Economist.

Barro, R. J. and D. Gordon, (1983), "Rules, discretion and reputation in a model of monetary policy", Journal of Monetary Economics, 12, pp. 101-121. 
Bernanke, B. S. and F. S. Mishkin, (1997), "Inflation targeting: A new framework for monetary policy ", NBER WP N 5893.

Calvo, G. and C. A. Végh, (1999), "Inflation stabilization and BOP crises in developing countries ", NBER WP $\mathrm{N}^{\circ} 6925$.

Carstens, A. G., D. C. Hardy and C. Pazabasioglu, (2004), "Avoiding banking crises in Latin America", Finance and Development, IMF, Septembre.

Cartelier, J. (1998), "Monnaie et comportements au marché : de l'interdépendance à la viabilité ", dans A. Vinokur (éd.), Décisions économiques, Paris, Economica, pp.129-144.

Cartelier, J. (2008), “ Money: the case for an alternative economic theory and beyond", Document de travail, Economix, Universite Paris $\mathrm{X}$-Nanterre.

Denizer, C., M. Dinç and M. Tarımcılar, (2000), "Measuring banking efficiency in the pre- and post-liberalization environment: Evidence from the Turkish banking system", The World Bank.

Edwards, S. and A. Cox-Edwards, (1987), Monetarism and Liberalization. The Chilean Experiment, Cambridge, MA, Ballinger.

Eichengreen, B. (2001), "Crisis Prevention and Management: Any New Lessons from Argentina and Turkey?”, Background paper for the World Bank's Global development Finance 2002.

Gürsel, S. (2007), "La Turquie : un marché de travail en transition ? ", Le 4 Pages du Centre d'Etudes de l'Emploi, N46, Septembre.

Hahn, F. H. (1984), Monnaie et inflation, Traduction française, Paris : Economica.

International Monetary Fund, Country Statistics, Différentes années. Keynes, J. M. (1965), A Treatise on Money, Macmillan.

Krueger, A. O. (1984), "Problems of Liberalization", in A. Harberger (ed.) World Economic Growth, San Francisco, ICS Press.

Lucas, R. E., (1972), "Expectations and the neutrality of money", Journal of Economic Theory, 4, pp. 103-124.

Marques-Pereira, J. et B. Théret, (2004), "Régimes politiques, médiations sociales et trajectoires économiques ", dans B. Lautier et J. Marques-Pereira (éd.), Brésil, Mexique. Deux trajectoires dans la mondialisation, Paris, Editions Karthala, pp. 25-83.

Marques-Pereira, J. (2006), Politique monétaire et droits sociaux : la souveraineté en question. Une hypothèse de comparaison de l'Argentine et $d u$ Brésil., Communication au Colloque 'Anthropologistes et économistes face à la globalisation', CLERSEIRD, Paris, 16-17 mars.

McCallum, B. T. (1997), "Crucial Issues Concerning Central bank Independence", Journal of Monetary Economics, 39, p.99-112.

Minsky, H. P. (1996), "Uncertainty and the Institutional Structure of Capitalist Economies", The Levy Economics Institute WP155.

Miotti, L. et D. Plihon, (2001), "Libéralisation financière, spéculation et crises bancaires ", Economie Internationale, 85, pp.3-36.

96 Economie et Institutions - n 10 et $11-1$ er \& 2e semestre 2007 
Orléan, A. (2006), "Monnaie, séparation marchande et rapport salarial", Document de travail.

Sargent, T. J. and N. Wallace, (1975), " 'Rational' expectations, the optimal monetary instrument, and the optimal money supply rule", Journal of Political Economy, 83, pp. 241-254.

Scialom, L. et Y. Zlotowski, (1994), "Les fondements institutionnels de la crise monétaire russe: un éclairage théorique ", Revue d'économie politique, 104(5), pp. 701-718.

Stiglitz, J. E. (1989), "Markets, Market Failures and Development", American Economic Review, Papers and Proceedings, 79(2), pp. 197203.

Stiglitz, J. E. (1999), "More instruments and broader goals: Moving toward Post-Washington Consensus", Revista de Economia Politica, 19 (1).

Théret, B. (2008a), "Présentation ", dans B. Théret (éd.), La monnaie dévoilée par ses crises, 2 volumes, Paris : Editions de l'EHESS.

Théret, B. (2008b), "De la monnaie considérée comme un fait social total ", Document de travail.

Ülgen, F. (1995), "Endogénéités de la monnaie. Fondements et définitions d'un concept: un bilan critique ", Economie Appliquée, 1995, N4, pp. 29-59.

Ülgen, F., H. Levent et S. Polat, (2003), "Le système bancaire turc : fragilités et crises ", Communication aux 7e rencontres euroméditerranéennes: Chocs exogènes, politiques d'ouverture et d'ajustement et négociations commerciales internationales dans la zone Euro-Méditerranéenne, Rabat, 8-10 octobre.

Ülgen, F. (2007), "Libéralisation et structures institutionnelles: transformations dans le système bancaire turc ", Colloque international Enjeux économiques, sociaux et environnementaux de la libéralisation commerciale des pays du Maghreb et du Proche-Orient, Organisation des Nations Unies, Commission économique pour l'Afrique-GATE, 19-20 octobre 2007, Rabat-Maroc.

Velasco, A. (1987), "Financial crises and balance of payments crises: A simple model of the Southern Cone experience ", Journal of Development Economics, 27(1-2), pp. 262-283.

World Bank (2006), Turkey. Country Economic Memorandum, WB, Expanded Report N³3549-TR, February 23, vol. II. 